\title{
The Optimal Aspect Ratio of Gold Nanorods for Plasmonic Bio-sensing
}

\author{
Jan Becker • Andreas Trügler • Arpad Jakab • \\ Ulrich Hohenester • Carsten Sönnichsen
}

Received: 9 October 2009 /Accepted: 1 February 2010/Published online: 2 March 2010

(C) Springer Science+Business Media, LLC 2010

\begin{abstract}
The plasmon resonance of metal nanoparticles shifts upon refractive index changes of the surrounding medium through the binding of analytes. The use of this principle allows one to build ultra-small plasmon sensors that can detect analytes (e.g., biomolecules) in volumes down to attoliters. We use simulations based on the boundary element method to determine the sensitivity of gold nanorods of various aspect ratios for plasmonic sensors and find values between 3 and 4 to be optimal. Experiments on single particles confirm these theoretical results. We are able to explain the optimum by showing a corresponding maximum for the quality factor of the plasmon resonance.
\end{abstract}

Keywords Plasmon · Sensors · Nanorods · BEM · Spectroscopy $\cdot$ Nanoparticles $\cdot$ Nanocrystals $\cdot$ Gold

\section{Introduction}

Gold nanoparticles have a long history as optical or electron microscopy labels. More recently, their plasmon resonance has been employed for more elaborate optical nanoscopicsensing schemes. The plasmon resonance of coupled particles depends on interparticle distance [1-4], and the

J. Becker · A. Jakab $\cdot$ C. Sönnichsen $(\square)$

Institute of Physical Chemistry, University of Mainz, Jakob-Welder-Weg 11,

55128 Mainz, Germany

e-mail: soennichsen@uni-mainz.de

A. Trügler $\cdot U$. Hohenester

Institute of Physics, Karl-Franzens University Graz,

Universitätsplatz 5,

8010 Graz, Austria strong plasmonic light scattering efficiency allows for the visualization of single nanorods, for example for orientation sensing [5-8]. Furthermore, the resonance position is influenced by the particle charge $[9,10]$ and the refractive index of the particle's immediate environment [11].

The plasmonic sensitivity to the immediate dielectric environment of the particles allows one to monitor the dielectric constant of liquids and binding events of molecules to the gold particle surface. Here, the nanoparticle sensor acts in a way similar to sensors exploiting the surface plasmon resonance on gold films, which is a standard method in many laboratories. However, whereas the detection scheme for surface plasmon resonance is usually a shift in the plasmon excitation angle [12], plasmonic nanoparticles show a shift in the plasmon resonance frequency [13]. The main advantage of using nanoscopic particles as sensors instead of metal films is their extremely small size which allows one, in principle, to measure analytes in volumes as small as attoliters [14]. The key factor for taking advantage of the small detection volume of plasmonic particles is the single particle plasmon-scattering spectroscopy [13, 15]. Single particle measurements probe the local environment around one specific particle, which - in principle - enables massive parallelization of nanoparticle plasmon sensors either for analyzing different analytes or obtaining statistics. Recently, we demonstrated a scheme that can conduct parallel sensing on randomly arranged nanoparticles using a liquid crystal device as an electronically addressable entrance shutter for an imaging spectrometer [16].

For such dielectric plasmon nanoparticle sensors, one hopes to have a large spectral shift for a given amount of analyte or refractive index change of the environment. Initially, spherical gold particles were used [14, 17] but were soon replaced by gold nanorods [18] due to their 
higher sensitivity on refractive index changes [19]. A lot of effort has been applied towards identifying the ideal plasmon sensor with a large spectral shift for a given change in refractive index, for example, using rod-shaped gold nanorattles [20], metamaterials [21], silver-coated gold nanorods [22] and others. However, rod-shaped nanoparticles remain popular for plasmonic applications. Some reasons for this are the ability to fabricate gold nanorods in high quality using seeded crystallization from solution, the adjustability of the plasmon resonance by varying the aspect ratio, the strong scattering efficiency, and the low plasmon damping in nanorods.

Here, by means of simulations and experiments, we investigate which aspect ratio (AR) of gold nanorods is ideal for plasmonic sensing by employing various measures for 'ideal' behavior. There are several different quantities that describe the performance of a plasmonic structure for sensing applications on a single particle level - and all of them have their merits for certain applications. We will discuss the most important of them in the following paragraphs - the plasmonic sensitivity to refractive index change as well as various 'figures of merit' - and present their dependency on nanorods' geometry from calculations for spherically capped gold rods with the boundary element method (BEM) [23, 24]. We confirm the identified trends by experimental results obtained using single particle dark field scattering spectroscopy.

\section{Plasmon sensor quality}

Changing the refractive index $n$ of the embedding medium by a given amount, $\mathrm{d} n$ shifts the plasmon resonance position in wavelength or energy units $\left(\lambda_{\text {res}}, E_{\text {res }}\right.$, respectively, see Fig. 1a). The corresponding proportionality constant or sensitivity $S_{\lambda}$ (often simply denoted $\Delta \lambda /$ RIU) can be expressed in wavelength $\left(S_{\lambda}\right)$ or energy $\left(S_{E}\right)$ units [25]: $S_{\lambda}=$ $\mathrm{d} \lambda_{\text {res }} / \mathrm{d} n$ and $S_{E}=d E_{\text {res }} / \mathrm{d} n=S_{\lambda} \mathrm{d} E / \mathrm{d} \lambda=-S_{\lambda} / \lambda^{2} \times 1,240 \mathrm{~nm} / \mathrm{eV}$.

The relatively broad plasmon linewidth $\Gamma$ (full width at half maximum) complicates the analysis further, because the plasmon linewidth of nanostructures with different geometries can vary more than tenfold. Since it is easier to detect a given resonance shift for narrow lines, the resonance shift relative to the linewidth is a more meaningful measure of the sensoric quality. This dimensionless quantity is often referred to as the 'figure of merit' $\mathrm{FOM}=S / \Gamma[26]$.

The FOM is easily determined experimentally and allows for the comparison of the plasmonic properties of many different structures with a single sharp plasmonic resonance. For more complex plasmonic responses (such as in metamaterial structures based on an analog of electromagnetically induced transparency (EIT) [21]), where the plasmon resonance does not follow a simple Lorentz peak shape, the concept needs to be refined. In practice, one would normally detect a spectral shift of a resonance as a relative intensity change $\mathrm{d} I / I$ at a fixed wavelength $\lambda_{0}$ induced by a small index change $\mathrm{d} n$. We can therefore define an alternative dimensionless figure of merit:

$\mathrm{FOM}^{*}=\left(\frac{(d I / d n)}{I}\right)_{\max }=\left(\frac{S_{\lambda}(d I / d \lambda)}{I}\right)_{\max }$

The wavelength $\lambda_{0}$ is chosen such that $\mathrm{FOM}^{*}$ has a maximum value - for gold nanorods in the shoulder of the resonance of the long-axis plasmon near the place where the slope $\mathrm{d} I / \mathrm{d} \lambda$ is highest.

Bio-sensing applications are even more complex. In this case, one seeks to detect the binding of small (organic) molecules to the nanoparticle surface instead of exchanging the entire embedding medium. The spectral shift now depends on the relative size of the molecules to the volume the plasmon field penetrates into the medium. Furthermore, the sensitivity is reduced with increasing distance to the particles surface. A 'figure of merit' trying to capture the different sensing volumes of various nanostructures can be defined as the $\mathrm{FOM}_{\text {layer }}{ }^{*}$ for a homogeneous coating of molecules with a specific refractive index (for example, $n=1.5$, typical for organic molecules) in a layer of thickness $l$ around the particle normalized to this layer thickness. The formal definition of this 'figure of merit for thin layers' $\mathrm{FOM}_{\text {layer }}{ }^{*}$ is therefore:

$\mathrm{FOM}_{\text {layer }}^{*}=\left(\frac{(d I / I)}{d l}\right)_{\text {max }}$

To compare the general sensing quality of different nanostructures, the limit of $\mathrm{FOM}_{\text {layer }}{ }^{*}$ for $l \rightarrow 0$ gives a defined value. Table 1 summarizes the different quantities used to determine the quality of plasmon sensors and their definitions.

\section{Results}

We simulate the light scattering cross-sections of gold nanorods by solving Maxwell's equations using the BEM and tabulated optical constants for gold [27]. Regarding shape, we use rods with spherical end-caps varying the particle length while keeping the diameter constant at $20 \mathrm{~nm}$. Even though the exact end-cap geometry influences the resonance position [28], many researchers have successfully used spherical end-caps for their simulations [29]. We vary the aspect ratio by changing only the particle length because gold nanoparticle synthesis usually results in particles of comparable width. From the BEM calculations, we find a linear relationship between aspect ratio AR and plasmon resonance wave- 


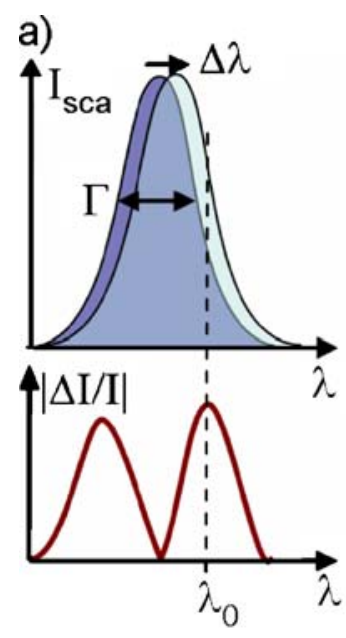

Fig. 1 a The plasmon resonance shifts $(\Delta \lambda)$ upon changing the refractive index of the surrounding medium by $\Delta n$. To detect such changes, the sensitivity $S_{\lambda}=\Delta \lambda / \Delta n$ needs to be large. Other important parameters that characterize plasmonic sensors are the plasmon linewidth $\Gamma$ and the wavelength $\lambda_{0}$ where the relative intensity change $|\Delta I / I|$ is largest (see text). b The plasmonic sensitivity in energy units

length: $\lambda_{\text {res }} / \mathrm{nm}=505+114$ (AR-1). The calculations of the layer effect for $\mathrm{FOM}_{\text {layer }}{ }^{*}$ were performed within the quasi-static approximation (QSA) for spheroids [30] since we did not implement coated particles in our BEM simulations so far. However, comparisons of QSA with full solutions for the Maxwell equations have shown good qualitative agreement [28].

The plasmon sensitivity in wavelength units $S_{\lambda}$ (calculated with BEM) shows the expected steady increase for an increasing aspect ratio [19] (not shown here), whereas the sensitivity in energy units $S_{E}$ shows a maximum at an aspect ratio of $\mathrm{AR}_{\max }=3$ (Fig. 1). Both the 'classical' FOM and the more general $\mathrm{FOM}^{*}$ show the same trend with two

Table 1 Summary of the different quantities describing the quality of plasmon sensors regarding their ability to detect changes in their environment: sensitivity $S_{\lambda}$ and $S_{E}$ in wavelength and energy units, the figures of merit as classical definition (FOM), in generalized form $\left(\mathrm{FOM}^{*}\right)$, and for thin layers $\left(\mathrm{FOM}_{\text {layer }}{ }^{*}\right)$

\begin{tabular}{llll}
\hline Quantity & Definition & $\mathrm{AR}_{\mathrm{opt}}$ & Max value \\
\hline$S_{\lambda}$ & $\mathrm{d} \lambda_{\text {res }} / \mathrm{d} n$ & $\infty$ & \\
$S_{E}$ & $d E_{\mathrm{res}} / \mathrm{d} n$ & $3.0(4.4)$ & $0.85 \mathrm{eV} / \mathrm{RIU}$ \\
$\mathrm{FOM}$ & $S_{\lambda} / \Gamma_{\lambda}=S_{E} / \Gamma_{E}$ & $4.3(3.2)$ & $11.1(10.9)$ \\
$\mathrm{FOM}^{*}$ & $(d I / I / d n)_{\max }$ & $4.2(3.1)$ & $24(23)$ \\
FOM $_{\text {layer }}{ }^{*}$ & $\lim _{l \rightarrow 0}\left(\frac{(d I / I)}{\mathrm{dl}}\right)_{\max }$ & $3.0(4.3)$ & $0.57 / \mathrm{nm}$ \\
\hline
\end{tabular}

The last columns list the optimal aspect ratio $\mathrm{AR}_{\mathrm{opt}}$ (the value in bracket corresponds to the second maximum) for gold nanorods with $20 \mathrm{~nm}$ diameter in an aqueous environment and the value of the corresponding quantity at the maximum (see "Results" and "Discussion" sections) maxima (Fig. 2a). The classical FOM has a maximum for rods with an aspect ratio of $\mathrm{AR}=4.3$ and a second local maximum at $\mathrm{AR}=3.2$. The maxima of the generalized $\mathrm{FOM}^{*}$ is slightly shifted to rod aspect ratios of $\mathrm{AR}=4.2 / 3.1$ (cf. Table 1). The values of the 'figure of merit for small layers' $\mathrm{FOM}^{*}$ layer (obtained by QSA calculations) are shown in Fig. 2b for increasing layer thickness $l$ and show maxima at the aspect ratios of $\mathrm{AR}=3.0$ and $\mathrm{AR}=4.3$. The first maximum at $\mathrm{AR}=3.0$ is higher for thin layers and in the limit of layer thickness $l \rightarrow 0$. Hence, rods with aspect ratios in the range of 3 to 4 are the best candidates to investigate changes in the refractive index of the embedding medium.

To verify the theoretical conclusions given above, we compared the theoretical results with experimental values measured on single particles. The particle spectra were obtained for nanorods immobilized on a glass substrate and exposed to liquids with various refractive indices in a dark field microscope coupled with an imaging spectrometer $[15,16]$. The resulting values are shown in Fig. 3 and show the same trend as predicted by the BEM simulations. The values are generally lower than the calculated values due to the influence of the supporting glass substrate and are potentially also influenced by a thin organic coating of the particles remaining from the synthesis. The variance within the experimental results is not a measurement error but originates from small derivations from the ideal particle geometry and environment. For example, defects in the nanoparticles' crystal structure would increase damping, therefore broadening the plasmon resonance and reducing FOM and FOM*. 

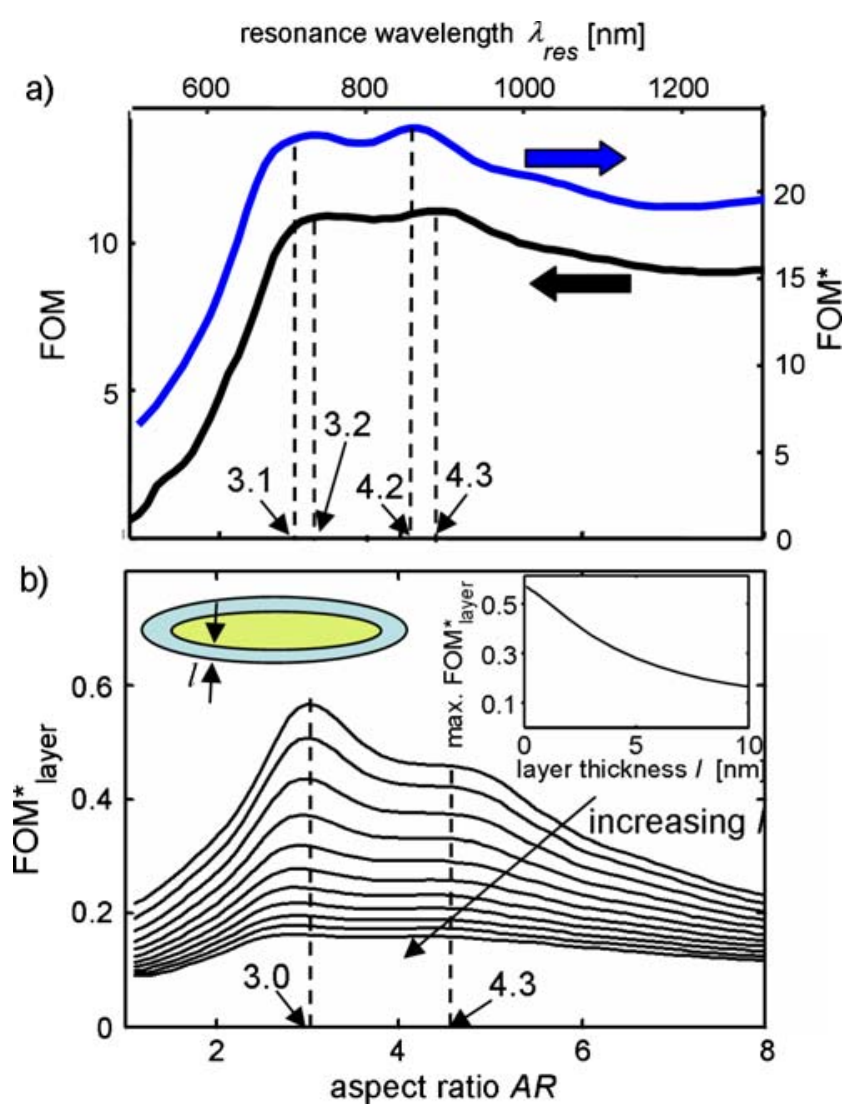

Fig. 2 To identify the optimal aspect ratio for plasmon sensing, various 'figure of merits' (FOM) are calculated a The classical FOM defined as plasmon sensitivity $S$ divided by plasmon linewidth $\Gamma$ (left axis) is compared to a generalized $\mathrm{FOM}^{*}$ defined as the maximal relative intensity change $|\Delta \mathrm{I} / \mathrm{I}|$ for a small refractive index change $\Delta n$ (right axis). While the classical figure of merit FOM has a maximum for an aspect ratio of $\mathrm{AR}=4.3$ and a second maximum at $\mathrm{AR}=3.2$, the generalized $\mathrm{FOM}^{*}$ has its maximum at $\mathrm{AR}=4.2$ with its second local maximum at $\mathrm{AR}=3.1$. b The $\mathrm{FOM}^{*}$ layer takes into account the sensing volume by considering the plasmon change induced by a small layer around the particle normalized to the layer thickness $(0.01 \mathrm{~nm}<1<10 \mathrm{~nm}$ in $1 \mathrm{~nm}$ steps). The plasmon sensing quality $\mathrm{FOM}^{*}{ }_{\text {layer }}$ decreases with increasing layer thickness (inset) and shows a maximal value of $\mathrm{FOM}^{*}{ }_{\text {layer }}=0.55$ for $\mathrm{d} \rightarrow 0$ at an aspect ratio $\mathrm{AR}=3.0$. (Data for a are calculated by BEM for gold nanorods with spherical end-caps and $20 \mathrm{~nm}$ diameter, data for $\mathbf{b}$ within the quasi static approximation QSA for spheroids.)

\section{Discussion}

The existence of a maximum of the plasmon sensitivity in energy units can be derived from a simple calculation of the polarizability of a spheroid in quasi-static approximation:

The polarizability $\alpha$ of a spheroid within the quasi-static approximation is given by [31]:

$\alpha=V \varepsilon_{0} \frac{\varepsilon_{r}-1}{1+L\left(\varepsilon_{r}-1\right)}$

where, $V$ is the particle volume, $\varepsilon_{r}=\varepsilon_{\text {particle }} / \varepsilon_{\text {medium }}$ is the relative dielectric function of the particle with respect to the medium, and $L$ is a shape factor. The exact equation for $L$ is given by [32]:

$$
L=\frac{1}{\mathrm{AR}^{2}-1} \cdot\left(\frac{\mathrm{AR}}{2 \cdot\left(\mathrm{AR}^{2}-1\right)^{1 / 2}} \cdot \ln \left(\frac{\mathrm{AR}+\left(\mathrm{AR}^{2}-1\right)^{1 / 2}}{\mathrm{AR}-\left(\mathrm{AR}^{2}-1\right)^{1 / 2}}\right)-1\right) .
$$

For $\mathrm{AR}<8$, this formula is simplified to $L \approx(1+\mathrm{AR})^{-1.6}$ with an error of $<5 \%$ [33].

Equating the real part of the denominator of Eq. 3 to zero and using the Drude model to describe the dielectric function of the particle $\left(\operatorname{Re}(\varepsilon) \approx \varepsilon_{\infty}-\omega_{\mathrm{p}}{ }^{2} / \omega^{2}[33]\right)$, the resonance position $E_{\text {res }}$, the plasmon sensitivity $S_{E}$, and the aspect ratio with the highest $S_{E}$ value $\mathrm{AR}_{\max }$ follow:

$$
E_{\mathrm{res}}=\frac{\omega_{p}}{\sqrt{n^{2} / L+\varepsilon_{\infty}-n^{2}}}
$$
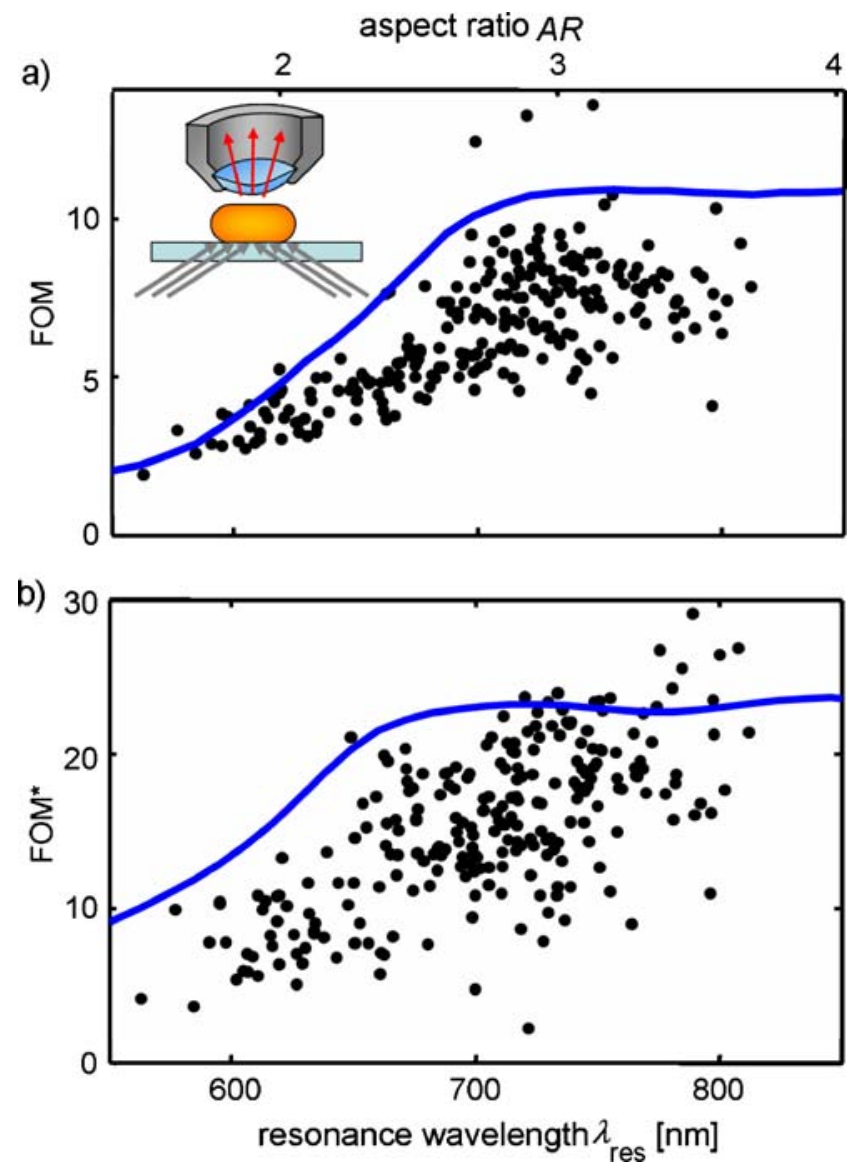

Fig. 3 Experimental data (each black dot represents one single particle) for the figures of merit FOM (a) and FOM* (b) obtained by single particle spectroscopy in a dark field microscope (inset). The absolute values are slightly lower than the theoretical prediction (blue line, see also Fig. 2a) due to the attachment of the gold rods to a glass substrate which limits the accessible surface (inset) 
$S_{E}=\frac{d}{\mathrm{dn}} E_{r e s}=\frac{\omega_{p} n(1 / L-1)}{\left(n^{2} / L+\varepsilon_{\infty}-n^{2}\right)^{3 / 2}}$

$\mathrm{AR}_{\max }=\left(\frac{n^{2}}{n^{2}+2 \varepsilon_{\infty}}\right)^{-1 / 1.6}-1$

where, $\omega_{p}$ and $\varepsilon_{\infty}$ are the plasma frequency of the conduction electrons ( $9 \mathrm{eV}$ for gold [34]) and the polarizability of the inner electrons (9.84 for gold [35]), respectively, and $n=\left(\varepsilon_{\text {medium }}\right)^{0.5}$ the medium's refractive index.

The agreement of these simple calculations with the BEM calculations is surprisingly good (Fig. 1 black lines) since the quasi-static approximation is only valid for spheroidally shaped particles much smaller than the wavelength of the incident light. The particles we investigate are neither spheroids nor much smaller than the light wavelength (only a factor of up to 5). Our BEM calculations using spherically capped cylinders model the real particle shape more realistically and correctly take the particles' absolute size into account.

Interestingly, the aspect ratio with the highest plasmon sensitivity in energy units $\mathrm{AR}_{\max }$ depends on the polarizability of the inner electrons of the metal $\varepsilon_{\infty}$ and the refractive index of the medium. For water $(n=1.33)$ as a medium, the optimal aspect ratio becomes $\mathrm{AR}_{\max }=3.8$. Since the Drude model does not include the contribution of interband damping to the imaginary part of the dielectric function, we conclude that the maximum in plasmonic sensitivity $S_{E}$ is not caused by the effect of those interband excitations.

Yet, what is the reason for the 'optimal aspect ratio' for the three 'figures of merit'? We believe the quality factor $Q$ is the key quantity in explaining this behavior. The quality factor of an oscillation describes the number of oscillations until the oscillation is damped. We find it plausible that a longer plasmon oscillation lifetime (i.e., a higher quality factor) results in a more sensitive dependency on changes in the surroundings. For a classical-driven harmonic oscillator, the resonance frequency divided by the linewidth of the resonance $\Gamma$ gives the quality factor $\left(Q=E_{\text {res }} / \Gamma\right)$. Figure 4 shows the quality factor as a function of the aspect ratio for gold nanorods (calculated by the BEM and measured experimentally on single particles). Evidently, a maximum is found around $A R=3$. The initial rise from $Q(A R=1)=8$ to $\mathrm{Q}(\mathrm{AR}=3)=22$ is easily explained considering the reduced plasmon damping as the resonance shifts to lower energies with an increasing aspect ratio [15]. The slow decrease above the aspect ratio of 3 is due to the decreasing resonance frequency divided by the (more or less) constant Drude damping.

The similarity between the way the quality factor and the figures of merit depend on the aspect ratio is a strong hint

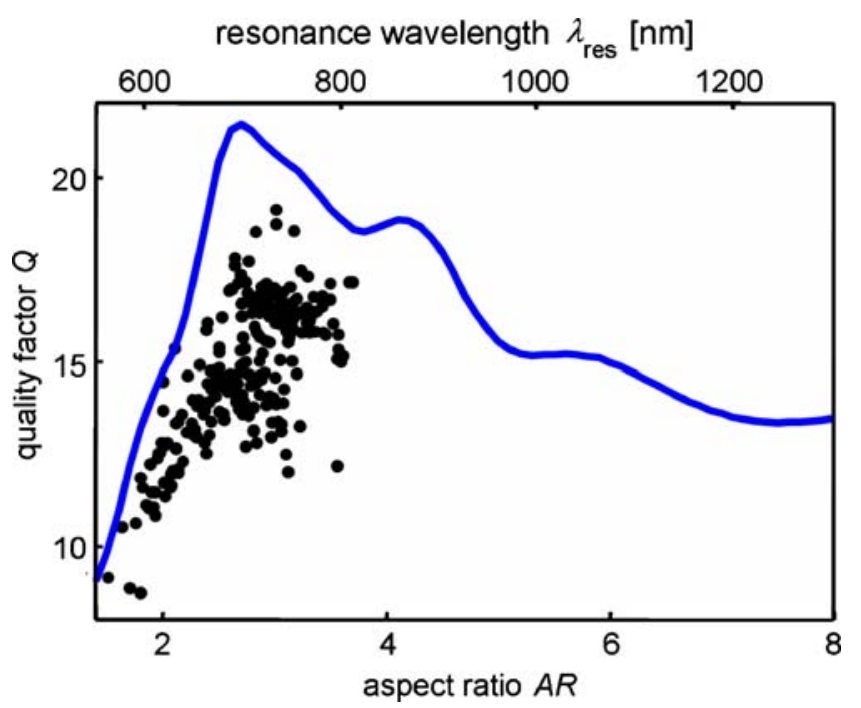

Fig. 4 The quality factor $Q$ (determined via $Q=E_{\text {res }} / \Gamma$ ) shows a maximum for rods with an aspect ratio of approximately $\mathrm{AR}=3$ and decreases for larger aspect ratios. The blue line represents the results obtained by BEM calculations, while the black dots correspond to measurements on single gold nanorods

but not solid proof that one is the cause of the other. It is desirable to have more detailed theoretical investigations of this aspect.

\section{Conclusion}

In summary, for the four measures for an 'optimal plasmonic sensor', namely sensitivity $S$, figure of merit FOM, generalized figure of merit $\mathrm{FOM}^{*}$, and figure of merit for thin layers $\mathrm{FOM}_{\text {layer }}{ }^{*}$, there is an optimum aspect ratio around 3-4, where gold nanorods show the best sensitivity. Only the plasmon sensitivity in wavelength units $S_{\lambda}$ increases steadily with aspect ratio. Contrary to many claims, the plasmonic properties of gold nanorods do not steadily increase towards the infrared spectral region but show an optimum in the near infrared. Even though gold nanorods show very strong plasmon resonances with high quality factors and are, at the same time, small in volume, we cannot exclude the idea that other shapes of metal structures (bi-pyramids, rattles, pairs of particles, EIT structures, etc) are even better plasmon sensors. We hope that our new definition of a generalized figure of merit and the corresponding generalized figure of merit for small layers will trigger the comparison of plasmon sensor quality of many more complicated metal structures.

Acknowledgment We acknowledge financial support by the DFG through the Emmy Noether Program (SO712/1-3), the MAINZ graduate school of excellence, and the Graz Advanced School of Science (NAWI GASS). 


\section{Appendix}

Methods

\section{Simulations based on boundary element method}

In our computational approach, we discretize the surface of the nanoparticle by a set of triangles and match the electromagnetic potentials at the triangle centers. By fulfilling the boundary conditions imposed by Maxwell's equations through auxiliary surface charges and currents, we end up with a generic and flexible scheme that allows us to compute the optical properties of arbitrarily shaped nanoparticles with complex geometry embedded in dielectric environments.

The dielectric function of the gold nanorods was extracted from optical data [27] and the chosen mesh sizes allow for a spatial resolution of approximately $1 \mathrm{~nm}$.

\section{Determination of S, FOM, FOM*, and FOM* ${ }_{\text {layer }}$}

We calculated the light scattering cross-section $C_{\text {sca }}$ for every particle shape (diameter $20 \mathrm{~nm}$, length from $20 \mathrm{~nm}$ to $160 \mathrm{~nm}$ in steps of $2 \mathrm{~nm}$ ) in $n_{1}=1.33$ and in $n_{2}=1.34$ as a function of wavelengths $\lambda$ (in steps of $1 \mathrm{~nm}$ ).

The FOM* $^{*}$ was calculated according to the following equation:

$\mathrm{FOM}^{*}=\left(\frac{\left|\operatorname{Csca}_{1}(\lambda)-\operatorname{Csca}_{2}(\lambda)\right|}{1 / 2 \times\left(\operatorname{Csca}_{1}(\lambda)+\operatorname{Csca}_{2}(\lambda)\right)}\right){ }_{\text {max }} /\left(n_{2}-n_{1}\right)$

The $\mathrm{FOM}^{*}$ layer was calculated in the same way in the quasi-static approximation for spheroids for layer thicknesses $l=0.01 \mathrm{~nm}$ to $10 \mathrm{~nm}$ in steps of $1 \mathrm{~nm}(0.01 \mathrm{~nm}$ until the first nanometer).

\section{Gold nanorods preparation}

Gold nanorods were synthesized according to the seeded growth procedure published by Nikoobakht et al. [36]. In this two-step synthesis, preformed seeds grow into rods in a concentrated surfactant solution. The samples used in this work were characterized by ensemble extinction spectroscopy and transmission electron microscopy to obtain the mean diameters and lengths of the different samples. The samples we used had the following properties:

\begin{tabular}{llrr}
\hline Name & $\lambda_{\text {res }}$ & Width & Length \\
LC 12-1 & 634 & $57 \pm 6$ & $28 \pm 4$ \\
S702 & 700 & $49 \pm 4$ & $18 \pm 2$ \\
S740 & 743 & $55 \pm 6$ & $18 \pm 3$ \\
\hline
\end{tabular}

Single particle spectroscopy

To investigate the spectral shift by changes in the refractive index of the environment on the single particle level, we dilute the nanorod suspensions 1:100 with distilled water and rinse them for $5 \mathrm{~min}$ through a flat glass capillary connected to PET tubing. Subsequent rinsing of $1 \mathrm{M}$ sodium chloride solution for 1 min can increase the number of immobilized particles. Afterwards, the glass capillary is rinsed for at least $15 \mathrm{~min}$ with distilled water $(n=1.333)$ to remove as many of the molecules as possible that were attached to the particle surface, and the scattering spectra of all particles in the field of view recorded. After rinsing the glass capillary with glucose solution $(25 \mathrm{wt} . \% n=1.372)$ for $15 \mathrm{~min}$, we again investigate the scattering spectra of the same particles and determine the quantities listed in Table 1.

\section{References}

1. Jain PK, Huang WY, El-Sayed MA (2007) On the universal scaling behavior of the distance decay of plasmon coupling in metal nanoparticle pairs: a plasmon ruler equation. Nano Lett 7 (7):2080-2088

2. Sönnichsen C, Reinhard BM, Liphardt J, Alivisatos AP (2005) A molecular ruler based on plasmon coupling of single gold and silver nanoparticles. Nat Biotechnol 23(6):741-745

3. Wang HY, Reinhard BM (2009) Monitoring simultaneous distance and orientation changes in discrete dimers of DNA-linked gold nanoparticles. J Phys Chem C 113(26):11215-11222

4. Reinhard BM, Siu M, Agarwal H, Alivisatos AP, Liphardt J (2005) Calibration of dynamic molecular rule based on plasmon coupling between gold nanoparticles. Nano Lett 5(11):2246-2252

5. Sönnichsen C, Alivisatos AP (2005) Gold nanorods as novel nonbleaching plasmon-based orientation sensors for polarized single-particle microscopy. Nano Lett 5(2):301-304

6. Bingham JM, Willets KA, Shah NC, Andrews DQ, Van Duyne RP (2009) Localized surface plasmon resonance imaging: simultaneous single nanoparticle spectroscopy and diffusional dynamics. J Phys Chem C 113(39):16839-16842

7. Pierrat S, Hartinger E, Faiss S, Janshoff A, Sonnichsen C (2009) Rotational dynamics of laterally frozen nanoparticles specifically attached to biomembranes. J Phys Chem C 113(26):11179-11183

8. Schubert O, Becker J, Carbone L, Khalavka Y, Provalska T, Zins I, Sönnichsen C (2008) Mapping the polarization pattern of plasmon modes reveals nanoparticle symmetry. Nano Lett 8 (8):2345-2350

9. Novo C, Funston AM, Mulvaney P (2008) Direct observation of chemical reactions on single gold nanocrystals using surface plasmon spectroscopy. Nat Nanotechnol 3(10):598-602

10. Carbone L, Jakab A, Khalavka Y, Sönnichsen C (2009) Lightcontrolled one-sided growth of large plasmonic gold domains on quantum rods observed on the single particle level. Nano Lett 9 (11):3710-3714

11. Perez-Juste J, Pastoriza-Santos I, Liz-Marzan LM, Mulvaney P (2005) Gold nanorods: synthesis, characterization and applications. Coord Chem Rev 249(17-18):1870-1901

12. Homola J, Yee SS, Gauglitz G (1999) Surface plasmon resonance sensors: review. Sens Actuators B Chem 54(1-2):3-15 
13. Sönnichsen C, Geier S, Hecker NE, von Plessen G, Feldmann J, Ditlbacher H, Lamprecht B, Krenn JR, Aussenegg FR, Chan VZH, Spatz JP, Moller M (2000) Spectroscopy of single metallic nanoparticles using total internal reflection microscopy. Appl Phys Lett 77(19):2949-2951

14. Raschke G, Kowarik S, Franzl T, Sönnichsen C, Klar TA, Feldmann J, Nichtl A, Kurzinger K (2003) Biomolecular recognition based on single gold nanoparticle light scattering. Nano Lett 3(7):935-938

15. Sönnichsen C, Franzl T, Wilk T, von Plessen G, Feldmann J, Wilson O, Mulvaney P (2002) Drastic reduction of plasmon damping in gold nanorods. Phys. Rev. Lett. 88(7):077402

16. Becker J, Schubert O, Sönnichsen C (2007) Gold nanoparticle growth monitored in situ using a novel fast optical single-particle spectroscopy method. Nano Lett 7(6):1664-1669

17. McFarland AD, Van Duyne RP (2003) Single silver nanoparticles as real-time optical sensors with zeptomole sensitivity. Nano Lett 3(8):1057-1062

18. Baciu CL, Becker J, Janshoff A, Sönnichsen C (2008) Proteinmembrane interaction probed by single plasmonic nanoparticles. Nano Lett 8(6):1724-1728

19. Lee KS, El-Sayed MA (2006) Gold and silver nanoparticles in sensing and imaging: sensitivity of plasmon response to size, shape, and metal composition. J Phys Chem 110(39):19220-19225

20. Khalavka Y, Becker J, Sönnichsen C (2009) Synthesis of rodshaped gold nanorattles with improved plasmon sensitivity and catalytic activity. J Am Chem Soc 131(5):1871-1875

21. Liu N, Weiss T, Mesch M, Langguth L, Eigenthaler U, Hirscher M, Sönnichsen C, Giessen H (2009) Planar Metamaterial Analogue of Electromagnetically Induced Transparency for Plasmonic Sensing. Nano Lett. doi:10.1021/n1902621d

22. Becker J, Zins I, Jakab A, Khalavka Y, Schubert O, Sönnichsen C (2008) Plasmonic focusing reduces ensemble linewidth of silvercoated gold nanorods. Nano Lett 8(6):1719-1723
23. Hohenester U, Krenn J (2005) Surface plasmon resonances of single and coupled metallic nanoparticles: A boundary integral method approach. Phys. Rev. B 72(19):195429

24. de Abajo FJG, Howie A (2002) Retarded field calculation of electron energy loss in inhomogeneous dielectrics. Phys. Rev. B 65(11): 115418

25. Burgin J, Liu MZ, Guyot-Sionnest P (2008) Dielectric sensing with deposited gold bipyramids. J Phys Chem 112(49):19279-19282

26. Sherry LJ, Chang SH, Schatz GC, Van Duyne RP, Wiley BJ, Xia YN (2005) Localized surface plasmon resonance spectroscopy of single silver nanocubes. Nano Lett 5(10):2034-2038

27. Johnson PB, Christy RW (1972) Optical Constants of the Noble Metals. Phys Rev B 6(12):4370-4379

28. Prescott SW, Mulvaney P (2006) Gold nanorod extinction spectra. c 99(12):123504

29. Bryant GW, De Abajo FJG, Aizpurua J (2008) Mapping the plasmon resonances of metallic nanoantennas. Nano Lett 8(2):631-636

30. Liu MZ, Guyot-Sionnest P (2004) Synthesis and optical characterization of $\mathrm{Au} / \mathrm{Ag}$ core/shell nanorods. J Phys Chem B 108 (19):5882-5888

31. Bohren CF, Huffman DR (1983) Absorption and Scattering of Light by Small Particles. Wiley

32. Osborn JA (1945) Demagnetizing factors of the general ellipsoid. Phys Rev 67(11-1):351-357

33. Sönnichsen C (2001) Plasmons in metal nanostructures. Cuvillier Verlag Göttingen

34. Lambrecht A, Pirozhenko I, Duraffourg L, Andreucci P (2007) The Casimir effect for silicon and gold slabs. Epl 77(4):44006

35. Cao M, Wang M, Gu N (2009) Optimized surface plasmon resonance sensitivity of gold nanoboxes for sensing applications. J Phys Chem C 113(4):1217-1221

36. Nikoobakht B, El-Sayed MA (2003) Preparation and growth mechanism of gold nanorods (NRs) using seed-mediated growth method. Chem Mater 15(10):1957-1962 